\title{
Introduction of HPV vaccination in Kenya'
}

\author{
Heleen Vermandere \\ Department of Uro-gynaecology, Faculty of Medicine and Health Science, \\ Ghent University
}

\begin{abstract}
Introduction: HPV vaccination prevents cervical cancer, the fourth most common cancer among women worldwide. Measured HPV vaccine acceptability is often high but does it also lead to high uptake?

Methodology: A cohort was set up assessing HPV vaccine acceptability and other health behaviour constructs before, and vaccine uptake after an HPV vaccination programme in Eldoret, Kenya. Focus groups shed light on the motivation for vaccine uptake or refusal.

Results: Acceptability was high but was no strong predictor of uptake, and neither were the constructs of the Health Belief Model. Lack of information and fear of side effects were major barriers. Feeling uncomfortable to discuss cervical cancer hampered open communication.

Discussion: Distrust towards new vaccines and the health system blocked translation from willingness-to-vaccinate to actual uptake, as did organizational factors such as poor promotion.

Conclusion: Future research should include broader concepts such as vaccine hesitancy and factors beyond personal control in order to predict vaccine uptake.
\end{abstract}

Key words: HPV vaccination, acceptability, uptake, longitudinal study, Kenya

\section{Introduction}

Cervical cancer poses a serious health threat to sexually active women, especially in low and middle income countries (LMIC) where screening opportunities are often low and treatment remains inaccessible for most of the affected women. Primary prevention through human papillomavirus (HPV) vaccination may therefore provide a turning point in the battle against cervical cancer (Denny et al., 20I4:I389-I398). However, prior to implementing large-scale vaccination programmes, several knowledge gaps need to be addressed. Therefore, formative research has been conducted measuring (hypothetical) acceptability and identifying potential barriers to successful implementation. In general, the vaccine is well accepted, with as main reported barriers costs and fear of side effects (Allen et al., 2010:4027-37).

The introduction of the HPV vaccine gave indeed rise to many studies gauging the public's perspectives, often applying health behaviour theories such as the Health Be-

This is the report of $\mathrm{PhD}$ research carried out at Ghent University, under the supervision of Olivier Degomme and Kristien Michielsen. 
lief Model (HBM) to identify determinants of acceptability (i.e. susceptibility and severity of cervical cancer, barriers and benefits of the vaccine, self-efficacy, and cues to action) (Brewer \& Fazekas, 2007:I07-I4). However, most of these studies took place in industrialized regions and did not measure HPV vaccine uptake. As such, several questions arise: I) Is the HPV vaccine acceptable in low-income countries, where it can have most impact, and are the determinants similar? 2) Does high acceptability always lead to high HPV vaccine uptake, i.e. the true outcome of interest? In order to answer these questions, a study with three objectives was set up.

\section{Objectives}

- Objective I: To determine the acceptability and subsequent uptake of the HPV vaccine in the context of an HPV pilot vaccination programme in Eldoret, Kenya.

- Objective 2: To ascertain whether health behaviour theories can be effectively applied to predict HPV vaccination in Eldoret, Kenya.

- Objective 3: To identify factors that influence successful introduction of an HPV vaccination programme in Eldoret, Kenya.

\section{Methodology}

A longitudinal study was rolled-out, with baseline and follow-up taking place before and after the implementation of an HPV vaccination effort. The programme freely offered the HPV vaccine to girl pupils of primary schools in Eldoret: the vaccine was administered in the referral hospital but promotion was done at school by teachers. A cohort of randomly selected mothers of these eligible girls was set up and invited to participate twice in a quantitative interview regarding cervical cancer prevention. HPV vaccine acceptability was measured at baseline while uptake got reported at follow-up. This design allowed studying acceptability and uptake, including the relation between these two main outcomes. In addition, a qualitative component was implemented at follow-up, evaluating the programme from the perspective of key-informants.

\section{Results}

The HPV vaccination programme took place from May 2012 until March 20I3. Baseline and follow-up data were collected two months before and after the programme, in March 2012 and May 2013, respectively. Of the 287 women interviewed in 2012, 89.2 percent (256/287) agreed to be interviewed again the next year. Also focus group discussions were organized in May 2013, with fathers (3), teachers (4) and vaccinators (I).

While acceptability of the HPV vaccine among the mothers was very high at baseline (88 percent accepted), only 3I percent had eventually vaccinated their daughter, and 5I percent reported that they had wanted to vaccinate but had missed the opportunity. Results showed that among this latter group, 55 percent had not received information regarding the whereabouts of the programme.

Finally, among those who had actively decided not to vaccinate (I8 percent), 42 percent mentioned fear of side effects as barrier and $3 \mathrm{I}$ percent said the partner opposed to 
vaccinating the daughter against cervical cancer. In general, the majority of the participants reported that unknown side effects made them doubt about HPV vaccination and that they needed more information in order to make an informed decision.

No strong relation was found between HPV vaccine acceptability and vaccination. Also with regard to the applicability of the Health Belief Model (HBM), no associations were found between the constructs and uptake, except for self-efficacy which positively influenced vaccination. The predictive value of the model did increase after adding a variable reporting whether or not the mother had received adequate information; being wellinformed led to higher vaccine uptake.

The qualitative data revealed that teachers and fathers had poor knowledge regarding cervical cancer and felt rather uncomfortable sharing information about it. This had also led to poor communication regarding the vaccination programme. Teachers also missed support of health care providers to address the questions of the parents as well as their own doubts. In general, HPV vaccination was not considered important given that cervical cancer was perceived as a consequence of a modern lifestyle with which the participants did not feel related. Finally, distrust towards (new) vaccines also hampered uptake: some thought it was an experiment while others had lost faith in vaccines during previous vaccination experiences. Suspicion did however fade away after a couple of months, once the community was convinced about the safety of the vaccine.

\section{Discussion}

HPV vaccine acceptability was not a strong predictor of vaccine uptake. The translation of intention into behaviour was mainly hampered because information about the vaccination programme did not reach all parents. Limited knowledge regarding HPV and cancer, and cultural taboos made it difficult for teachers and parents to discuss HPV vaccination (Perlman et al., 20I4). Therefore, direct communication of health care providers with caregivers seems crucial as the former is better posted to discuss such topics. Moreover, failure of achieving such a contact moment did not only lead to poor uptake but also allowed the spread of rumours and doubts. Future programmes will need to have more attention for two-way communication, to address misbeliefs, as opposed to the top-down approach usually used.

So while HPV vaccine uptake was lower than expected (LaMontagne et al., 20II:82I$830 \mathrm{~B})$, mainly due to organizational reasons, HPV vaccine acceptability reported at baseline might have been overestimated because of several factors. First of all, given that awareness regarding cervical cancer and HPV vaccination is generally low in Kenya, some participants were probably overtaken by the new information which interfered with their estimation of intention, tending to overvalue (also partly due to social desirability). In addition, people may overestimate their intentions - especially since new behaviours barriers are hard to predict - or express a desire rather than a true plan of action. Finally, given the novelty of HPV vaccination, it is not always clear who will take the final decision and as reported by the women in the quantitative study, in the end many fathers had an important role in the decision-making. As a result, women were not in control of their 
behaviour meaning that their intentions were no longer relevant as a predictor for the vaccination status of the daughter (Sheeran, 20II:I-36).

Taken all together, the poor vaccine uptake, due to promotional flaws, combined with the high reported acceptability led to an imbalance in the relation acceptability-uptake which was mainly driven by low acceptability leading to non-uptake, rather than by acceptors fulfilling their own reported objectives.

Similar to acceptability, also the HBM constructs were poor determinants of uptake (Gerend \& Shepherd, 20I2:I7I-80). Given that these measurements only assess personal beliefs and attitudes, and given the above-mentioned importance of programmatic strategies, adding variables beyond the personal perspective, at the organizational level, might improve the predictive value of the theory. In addition, also the value of each of the constructs can be questioned. Regarding the absence of a correlation of uptake with severity and susceptibility, this is not so surprising given the Kenyan context: access to high quality care is limited and diseases often remain untreated which makes them more lifethreatening. Especially (cervical) cancer is considered deadly. As such, perceiving cervical cancer as a threat is common and, consequently, does not distinguish those who vaccinate against HPV and those who do not. With regard to foreseen benefits and barriers, one can wonder how reliable people's estimates can be regarding the advantages and disadvantages of a new, unknown vaccine. By measuring users' and non-users' perspectives, more realistic determinants might be identified. Also trust in the vaccine or in the health system might be a stronger predictor, given that people rely on past, medical experiences. Also self-efficacy, which was positively associated with vaccine uptake, can be considered as trust, in oneself, to perform the desired behaviour (Carpenter, 2010:66I-669).

By focusing promotion on increasing trust, but also by choosing a delivery platform that instils confidence and convenience, vaccine uptake could thus potentially increase. A carefully designed programme should indeed take into account vaccine confidence, complacency and convenience and address them by both tailored promotion and adequate vaccine delivery. In the context of the HPV vaccination programme in Eldoret however, none of the three C's seemed to be properly fulfilled (Larson et al., 2014: 2150-59): some people distrusted the vaccine and the health providers in the referral hospital, cervical cancer was not always seen as a threat (whereas the vaccine had potential side effects) and offering the vaccine in a health centre was not considered the most convenient approach. School-based vaccination seemed more preferred but good cooperation between health staff and the teachers corpse will be crucial to obtain good coverage (Paul \& Fabio, 20I4:320-326).

\section{Conclusion}

Measuring HPV vaccine acceptability and its determinants is not sufficient to predict uptake. More attention should go to other important factors such as vaccine confidence or more broadly, vaccine hesitancy. In addition, variables beyond personal control contribute significantly to vaccine uptake pointing out the need to complement formative research - mostly conducted prior to implementation and based on personal health behaviour theories - with monitoring vaccination programmes and assessing users' and non-users' perspectives. 


\section{References}

Allen, J. D., Coronado, G. D., Williams, R. S., Glenn, B., Escoffery, C., Fernandez, M., \& Mullen, P. D. (2010). A systematic review of measures used in studies of human papillomavirus (HPV) vaccine acceptability. Vaccine, 28(24), 4027-4037.

Brewer, N. T., \& Fazekas, K. I. (2007). Predictors of HPV vaccine acceptability: a theory-informed, systematic review. Preventive medicine, 45(2), I07-II4.

Carpenter, C. J. (2010). A meta-analysis of the effectiveness of health belief model variables in predicting behavior. Health communication, 25(8), 66r-669.

Denny, L., Adewole, I., Anorlu, R., Dreyer, G., Moodley, M., Smith, T., \& Ramakrishnan, G. (20I4). Human papillomavirus prevalence and type distribution in invasive cervical cancer in sub-Saharan Africa. International journal of cancer, $134(6)$, I389-1 398.

Gerend, M. A., \& Shepherd, J. E. (2012). Predicting human papillomavirus vaccine uptake in young adult women: Comparing the health belief model and theory of planned behavior. Annals of Behavioral Medicine, 44(2), I7I-I80.

LaMontagne, D. S., Barge, S., Thi Le, N., Mugisha, E., Penny, M. E., Gandhi, S., \& Paul, P. (20II). Human papillomavirus vaccine delivery strategies that achieved high coverage in low-and middle-income countries. Bulletin of the World Health Organization, 89(II), 82I-830.

Larson, H. J., Jarrett, C., Eckersberger, E., Smith, D. M., \& Paterson, P. (20I4). Understanding vaccine hesitancy around vaccines and vaccination from a global perspective: a systematic review of published literature, 2007-2012. Vaccine, 32(19), 2150-2159.

Paul, P., \& Fabio, A. (2014). Literature review of HPV vaccine delivery strategies: Considerations for school-and nonschool based immunization program. Vaccine, 32(3), 320-326.

Perlman, S., Wamai, R. G., Bain, P. A., Welty, T., Welty, E., \& Ogembo, J. G. (20I4). Knowledge and awareness of HPV vaccine and acceptability to vaccinate in sub-Saharan Africa: a systematic review. PloS one, 9(3), egogi2.

Sheeran, P. (2002). Intention-behavior relations: A conceptual and empirical review. European review of social psychology, I2(I), I-36. 